\title{
Drawing on emotions: the evolving role of art therapy
}

\author{
Chelsea Cheng ${ }^{1, *}$, Mohamed Elhassan Elamin ${ }^{1,2}$, Helen May $^{2}$ and Miriam Kennedy ${ }^{1,2}$ \\ ${ }^{1}$ School of Medicine, Royal College of Surgeons in Ireland, Dublin, Ireland \\ ${ }^{2}$ Department of Psychiatry, Highfield Healthcare, Dublin, Ireland
}

\begin{abstract}
Art therapy is a form of psychotherapy that uses art media to improve well-being. This article explores the history and development of art therapy, from the first description of art therapy in the 1940s, to the two main approaches still in use today. The benefits of art therapy with regard to its delivery, patient population, and cost are then broadly discussed. The use of art therapy in psychiatric conditions, specifically in schizophrenia and schizophrenia-like conditions, is highlighted, as well as its role in chronic and physical conditions like asthma and cancer. Furthermore, the potential for art therapy to be more broadly implemented using technological novelties, such as virtual reality, is considered, especially in light of the COVID-19 pandemic.
\end{abstract}

Received 16 October 2020; Accepted 18 February 2021

Key words: Art therapy, psychotherapy, therapeutics, schizophrenia, patient care, rehabilitation, virtual reality, COVID-19.

\section{History and development of art therapy}

Art therapy was first described in the 1940s by Adrian Hill, an artist and author who recognized the important therapeutic role of art-making during his recovery from tuberculosis at King Edward VII Sanatorium in England (Bitonte et al., 2014; Case and Daley 2014; Hogan 2001). He believed that the act of creating art engaged a person fully, allowing diversion of attention from their troubles, and encouraged other patients to participate in painting with him (Case and Daley 2014; Hogan 2001). However, in addition to being a diversionary tactic, Hill also saw the process of creating art as a way to uncover and rid oneself of internal emotional conflict (Hogan 2001). Since then, Hill's concept of art therapy has continued to grow and develop, culminating in the present-day field of art therapy.

Art therapy has several different forms, but in broad terms, it is a type of psychotherapy that encourages expression and healing through art media and may be used to explore the relationships between the created art piece, the patient, and the therapist (American Art Therapy Association 2017; Case and Daley 2014). There are two main strands of art therapy. First of all, there is the use of art as therapy, which is the idea that the process itself of making art is healing, as originally described by Hill (Case and Daley 2014). The second strand of art therapy, based on Margaret Naumberg's work, involves applying aspects of psychoanalytic theory to explore transference and countertransference in a three-way relationship between the artwork, the patient, and the therapist (Case and Daley 2014; Schaverien 1999). In this

*Address for correspondence: Ms. Chelsea Cheng, BScH, School of Medicine, Royal College of Surgeons in Ireland, Dublin, Ireland. (Email: chelseacheng@rcsi.com) approach to art therapy, also known as analytical art psychotherapy, the patient's work is examined together with the therapist to better understand the patient's unconscious mind as manifested in their artwork (Case and Daley 2014; Schaverien 1999).

Although the ambiguity in the definition of art therapy may appear disadvantageous, in reality, its scope allows it to be adapted for a wide variety of uses and attuned to each patient's needs.

\section{Benefits of art therapy}

There are many benefits to using art therapy when compared to other forms of therapy. It is flexible in that it may be conducted one-on-one or in larger groups, depending on individual needs (British Association of Art Therapists 2020; Case and Daley 2014). It is suitable for all ages, from children to the elderly (British Association of Art Therapists 2020; Case and Daley 2014). Starting from as early as 15 months, children can begin to pick up a pencil and scribble (Ireland's National Childcare Directory 2020). By the age of 4 years, a child may already be able to draw a human figure (Ireland's National Childcare Directory 2020). Thus, art therapy may be applied even in very young children. Furthermore, art therapy focuses on the meaning of the artwork and the creation process itself, rather than the quality of art produced (Case and Daley 2014). Therefore, artistic ability need not be present, as no artistic skill is required in art therapy. As well, art can be created with minimal and inexpensive materials, for instance, with something as basic as printing paper and pencils, thereby foregoing the need to purchase expensive tools and equipment. However, the true potential of art therapy lies in its ability to be used in a wide variety of conditions, including both mental and physical illnesses. 


\section{Art therapy for psychiatric conditions}

Art therapy has been shown to be beneficial in the treatment of psychiatric conditions, including schizophrenia and schizophrenia-related disorders (Green 1987; Morrow 1985; Richardson et al. 2007; Teglbjaerg 2011), mood disorders (Bell and Robbins 2007; De Petrillo and Winner 2005), and even trauma (Schouten et al. 2014). Art therapy is particularly useful in situations where a patient is unable to fully express themselves verbally, as the art medium provides an alternative method of expression that does not rely on words (American Art Therapy Association 2017; Morrow 1985). This situation was seen in Patient $X$, a young adult with a working diagnosis of schizotypal disorder. Patient $X$ demonstrated the characteristic features of schizotypal disorder, including eccentric behavior, magical thinking, social anxiety, and unusual perceptual experiences, such as olfactory hallucinations (ICD-10 Online Version 2019). They also described another world which they frequently visited and would draw the characters that they saw. In Patient X's case, art therapy provided a means through which they could express themselves. They struggled with verbally describing the characters in their world, but was encouraged to bring their sketchbook along with multidisciplinary meetings, where they were able to present their world to the staff members. This improved the relationship between Patient $X$ and the consultant and allowed them to open up about their home life and their struggles with continuing college. Although the specific use of art therapy in schizotypal disorder has not been thoroughly researched, it has been applied successfully in associated clinical disorders, for example, in the treatment of schizophrenia (Green 1987; Morrow 1985; Richardson et al. 2007; Teglbjaerg 2011). In a randomized controlled trial conducted by Richardson et al. (2007), the use of group art therapy as an adjunctive therapy in patients with schizophrenia led to a statistically significant decrease in negative symptoms when compared to the use of standard psychiatric care. Moreover, in a qualitative study carried out by Teglbjaerg (2011), participation in art therapy resulted in an increased sense of self in patients with schizophrenia. Through engaging in the creative artmaking process and reflecting on the resulting art piece, patients reported decreased paranoia and anxiety and improved self-esteem (Teglbjaerg 2011). Consequently, there may be a role for art therapy in the treatment of schizophrenia and schizophrenia-related disorders.

\section{Art therapy for chronic and physical conditions}

In addition to the use of art therapy in psychiatric conditions, its use has also been shown to be beneficial in chronic, physical conditions (Bar-Sela et al. 2007; Beebe et al. 2010; Hwu 1995). When a patient is diagnosed with a physical illness, one does not typically think about the psychological effects that come along with the diagnosis; however, chronic physical illnesses often have large detrimental effects on psychological functioning (Hwu 1995). Art therapy may be provided in these cases to help patients manage their fears and improve their quality of life. For instance, a randomized controlled trial investigating the use of art therapy in children with asthma found that after a 7-week period of once-weekly art therapy sessions, children reported decreased anxiety and improved self-concept (Beebe et al. 2010). These positive effects were seen to persist at 6 months following the conclusion of the study (Beebe et al. 2010). Not only can art therapy be beneficial for children with chronic illnesses but it can also improve psychological outcomes in adult patients undergoing painful treatments. In a study by Bar-Sela et al. (2007), cancer patients undergoing chemotherapy who received an art therapy intervention had significantly improved depression scores. Patients also experienced a reduction in fatigue, a frequent symptom in those undergoing chemotherapy (Bar-Sela et al. 2007). Accordingly, the adjuvant use of art therapy in treating chronic illnesses may bring about positive outcomes for both children and adults.

\section{The future of art therapy}

With the announcement of the COVID-19 pandemic by the World Health Organization on 11 March 2020, many schools, businesses, and even services had to be shut down. Nonetheless, the rapid technological advances in recent years have provided a means through which services could be continued to be delivered online and virtually. Art therapy is one such service that has the potential to progress through technology. Developments in virtual reality (VR), digital art tools, and teletherapy may all contribute to the further advancement of this form of psychotherapy (American Art Therapy Association 2020). In particular, VR may offer participants the opportunity to partake in an art experience that enhances creativity and goes beyond the limits posed by the physical world (Austin 2009; Hacmun et al. 2018; Kaimal et al. 2019; Lohrius and Malchiodi 2018). For example, in a qualitative study conducted by Kaimal et al. (2019), participants reported an improvement in energy and motivation and an ability to create art that would otherwise not exist in the physical world. Moreover, it was noted that VR could be beneficial for patients who were isolated from others, either due to illness, disabilities, or age (Kaimal et al. 2019). Thus, this form of art therapy delivery has the potential to be used both in the current pandemic and in the future to improve reach and increase accessibility. 


\section{Conclusion}

To conclude, although art therapy is a newer form of psychotherapy, it is a beneficial intervention with a growing evidence base that supports its use in various patient populations. As art therapy continues to evolve and incorporate new technologies such as VR, there is the possibility for it to be more broadly implemented, particularly during the current COVID-19 pandemic where social distancing measures and lockdowns prevent in-person sessions. Therefore, art therapy is a promising strategy that should be considered for wider utilization by mental health services.

\section{Acknowledgements}

The authors would like to thank Highfield Healthcare, the Royal College of Surgeons in Ireland, Professor Vincent Russell, and the Irish Journal of Psychological Medicine for their support, comments, and suggestions.

\section{Conflict of interest}

The authors have no conflict of interest to declare.

\section{Ethical standards}

The authors assert that all procedures contributing to this work comply with the ethical standards of the relevant national and institutional committee on human experimentation with the Helsinki Declaration of 1975, as revised in 2008. The authors assert that ethical approval was not required for the publication of this manuscript.

\section{Financial supports}

This research received no specific grant from any funding agency, commercial or not-for-profit sectors.

\section{References}

Austin BD (2009). Renewing the debate: digital technology in art therapy and the creative process. Art Therapy: Journal of the American Art Therapy Association 26 (2), 83-85.

Bar-Sela G, Atid L, Danos S, Gabay N, Epelbaum R (2007). Art therapy improved depression and influenced fatigue levels in cancer patients on chemotherapy. PsychoOncology 16 (11), 980-984.

Beebe A, Gelfand EW, Bender B (2010). A randomized trial to test the effectiveness of art therapy for children with asthma. Journal of Allergy and Clinical Immunology 126 (2), 263-266.

Bell CE, Robbins SJ (2007). Effect of art production on negative mood: a randomized, controlled trial. Art Therapy 24 (2), 71-75.
Bitonte RA, De Santo M (2014). Art therapy: an underutilized, yet effective tool. Mental Illness 6 (1), 5354.

Case C, Daley T (2014). The Handbook of Art Therapy, 3rd ed., pp. 1-12. East Sussex: Routledge, Taylor \& Francis Group.

COVID-19 Resources for Art Therapists - American Art Therapy Association (2020). (https:/ /arttherapy.org/ covid-19-resources/). Accessed 1 September 2020.

Definition of Art Therapy - American Art Therapy Association (2017). (https://arttherapy.org/about-arttherapy/). Accessed 30 August 2020.

De Petrillo L, Winner E (2005). Does art improve mood? A test of a key assumption underlying art therapy. Art Therapy 22 (4), 205-212.

Green BL, Wehling C, Taisky GJ (1987). Group therapy as an adjunct to treatment for chronic outpatients. Psychiatric Services 38 (9), 988-991.

Hacmun I, Regev D, Salomon R (2018). The principles of art therapy in virtual reality. Frontiers in Psychology $\mathbf{9}$, 2082.

Hogan, S (2001). Healing Arts, pp. 132-156. London: Jessica Kingsley Limited.

Hwu Y-J (1995). The impact of chronic illness on patients. Rehabilitation Nursing 20 (4), 221-225.

ICD-10 Online Version - World Health Organization (2019). (https://icd.who.int/browse10/2019/en). Accessed 30 August 2020.

Kaimal G, Carroll-Haskins K, Berberian M, Dougherty A, Carlton N, Ramakrishnan A (2020). Virtual reality in art therapy: a pilot qualitative study of the novel medium and implications for practice. Art Therapy 37 (1), 16-24.

Lohrius J, Malchiodi C (2018). The Handbook of Art Therapy and Digital Technology, pp. 215-229. Philadelphia, PA: Jessica Kingsley Publishers.

Morrow R (1985). The use of art therapy in a patient with chronic schizophrenia. Jefferson Journal of Psychiatry 3 (1), 48-51.

Richardson P, Jones K, Evans C, Stevens P, Rowe A (2007). Exploratory RCT of art therapy as an adjunctive treatment in schizophrenia. Journal of Mental Health 16 (4), 483-491.

Schaverien J (1999). The Revealing Image, pp. 13-25. London: Jessica Kingsley Publishers.

Schouten KA, De Niet GJ, Knipscheer JW, Kleber RJ, Hutschemaekers GJM (2014). The effectiveness of art therapy in the treatment of traumatized adults. Trauma, Violence, \& Abuse 16 (2), 220-228.

Scribble - Ireland's National Childcare Directory (2020). (https://www.childcare.ie/news/scribble-62). Accessed 2 September 2020.

Teglbjaerg HS (2011). Art therapy may reduce psychopathology in schizophrenia by strengthening the patients' sense of self: a qualitative extended case report. Psychopathology 44 (5), 314-318.

What is Art Therapy? - British Association of Art Therapists (2020). (https: / / www.baat.org/About-ArtTherapy\#). Accessed 30 August 2020. 